\title{
An intelligent PET interface for environmental control and data acquisition and analysis
}

\author{
EDWARD K. CROSSMAN, MICHAEL R. STEPHENSON, and HAROLD L. LYNCH \\ Utah State University, Logan, Utah 84322
}

\begin{abstract}
An interface, controlled by a Z- 80 central processor, that plugs into the PET microcomputer's IEEE-488 port is described. This interface is capable of controlling 48 separate $28-\mathrm{V}$ dc output signals, six of which are high current. Sixteen inputs (switch closures) can be accepted by the interface. Data tagged with real-time information are output automatically to cassette tape in PET format. This PET interface system is designed to accommodate a single experimental station.
\end{abstract}

\section{BACKGROUND}

The psychology animal laboratory at Utah State University consists of a variety of animal environments for studying both operant and classical conditioning. Typically, electromechanical or some mixture of electromechanical and minicomputer (PDP-8 family) equipment has been used to control experimental events and to record the resulting behavior changes. Recently, Crossman and Williams (1978) described a multiuser on-line microcomputer system for accomplishing these functions. This system, based on the Imsai 8080 microcomputer, was designed to accommodate up to five separate experiments occurring simultaneously.

The Imsai system was based on the concept of a single, centralized computer sharing the power and functions of its central processor with multiple experimental stations, each of which could have distinct requirements. This concept was born of economic necessity in an era when minicomputers were priced at $\$ 10,000$ or more. The basic problem with the central, master computer concept is that failure of the master computer forces the shut-down of all its attached stations. Moreover, the programs required to keep track of all the events simultaneously happening in the satellite environments are unduly complicated by the limitation of a single central processor.

Fortunately, the costs of integrated circuits (chips) have continued to decline, owing to improvements in manufacturing, increased competition, and demand. Relatively powerful computers such as the Radio Shack TRS- 80 or the Commodore PET can be purchased for less than $\$ 800$, making it unnecessary to spread the capabilities of a single computer across multiple concurrent demands. Rather, it is now possible, and desir-

This research was supported by Grant IP 1104 from the Utah State University Division of Research to Edward $K$. Crossman. Requests for reprints should be addressed to Edward K. Crossman, Department of Psychology, Utah State University, Logan, Utah 84322. able, to dedicate a single microcomputer to a single experimental station. Failure by one such computer only forces one station to cease operation until a substitute computer can be plugged in. The inoperable computer can then be repaired without interrupting valuable experimental progress.

Such microcomputers are designed to accomplish a wide variety of tasks, but not specifically those required by an experimental psychology laboratory. In the lab. oratory, it is necessary to interface the computer and the test station.

This paper describes an interface that allows the PET computer to control experimental events and to record subject behavior on cassette tape for subsequent data analysis. The term intelligent interface refers to an interface that has its own central processing unit (CPU), which greatly increases the capabilities of the interface, compared with one that lacks a CPU. McLean (1978) describes a simple interface for the PET and provides a description of the PET's features. The brief description of the PET offered here concentrates only on those features relevant to the intelligent interface.

\section{THE PET}

The PET microcomputer comes in various models with memory sizes ranging from $4 \mathrm{~K}$ to $32 \mathrm{~K}$ bytes of read and write (RAM) memory. Any PET model works with the interface described here. The PET has a 9-in. videoscreen, a keyboard, and a cassette recorder as the primary means of loading or saving programs or data. Peripheral devices currently available from Commodore Business Machines, Inc., manufacturer of the PET, include an impact printer and a floppy disk unit for mass storage.

The PET has four connectors by which communication with the external world can be achieved: (1) an 8-bit parallel port, (2) a connector for a second cassette recorder, (3) a memory expansion connector for additional memory, and (4) an IEEE-488 connector for attaching a wide variety of laboratory instruments to 
the PET. Because this connector permits extensive handshaking capabilities (the ability to carefully regulate the flow of information), it is the connector by which the intelligent interface described below is attached to the PET.

The language used by the PET is BASIC, and it permanently resides in read-only memory (ROM) in the form of a BASIC interpreter. It is a relatively easy language to learn and is easily adapted to accomplish the sort of on-line process control that interests psychologists. Although interpreters are somewhat slow, by dedicating a single PET to a single experimental station, this slowness is not a significant disadvantage.

Finally, unlike many currently available microcomputers, the PET contains an internal real-time clock with $1 / 60 \mathrm{sec}$ (a jiffy) resolution as standard equipment. This clock is incremented automatically by the PET and can be read at any time by the user in the form of six digits representing hours, minutes, and seconds or as six digits representing the number of jiffies.

\section{INTELLIGENT INTERFACE}

\section{General Description}

The purpose of the project described here was to design an interface between the PET computer and an animal or human experimental station (see Figure 1). The interface had to have three functions: (1) to control experimental events (stimulus displays, reinforcement deliveries, etc.) in a $28-\mathrm{V} \mathrm{dc}$ environment, (2) to acquire digital data in real-time, such as switch closures, and (3) to store data on cassette tape in PET format such that any PET computer could subsequently read and analyze the data.

\section{Hardware Description}

Figure 2 shows in block format the essential components of the interface. The Z-80 CPU, running at $4 \mathrm{MHz}$, directs the flow of data and controls information between the PET's IEEE-488 bus and the external world through the interface's parallel (PIO) port. This port is represented by screw-type barrier strips to which are attached wires that terminate at the user's end in $\mathrm{Nu}$ Way-type snaps. These snap leads go directly to whatever electromechanical modules the user has, such as a chamber controller panel and a visual controller panel (for use with in-line displays). Because the interface

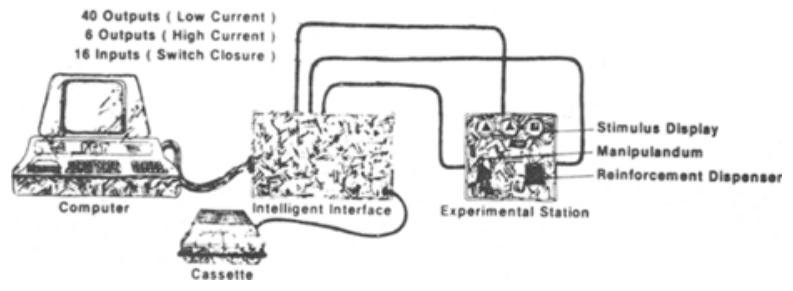

Figure 1,

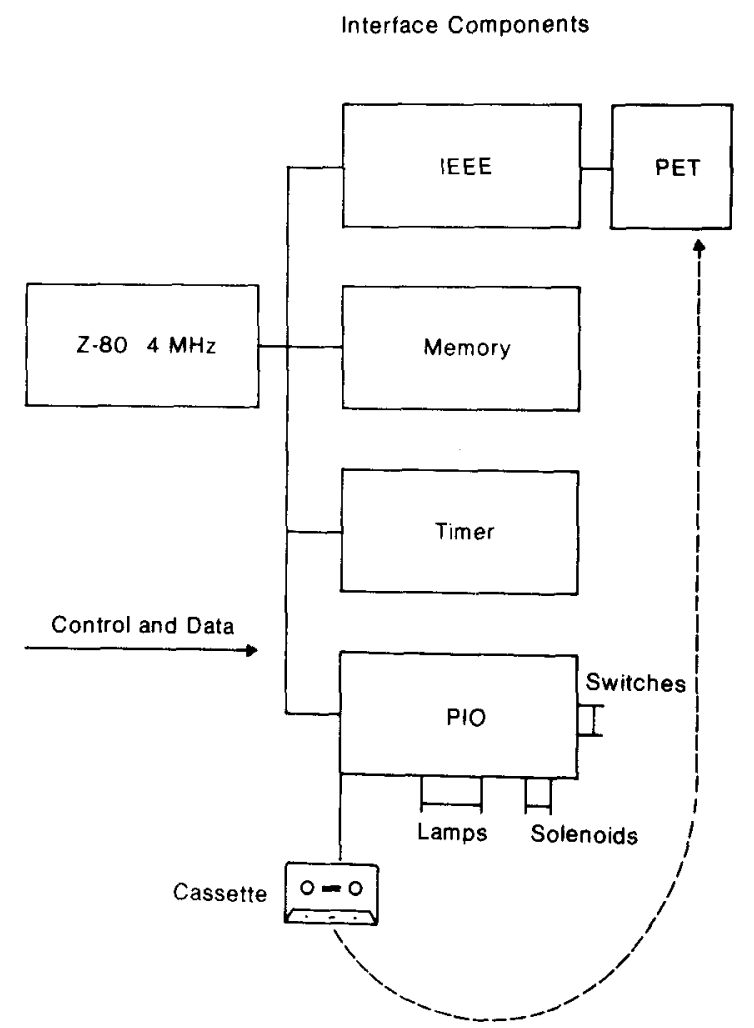

Figure 2.

contains a $28-\mathrm{V} \mathrm{dc}$ power supply, the signals represented at the port are $28 \mathrm{~V}$. Specifically, the interface supplies 42 separate low-current (up to $200 \mathrm{~mA}$ at $28 \mathrm{~V} \mathrm{dc}$ ) outputs for lamps and 6 high-current (up to $2 \mathrm{~A}$ at $28 \mathrm{~V} \mathrm{dc}$ ) outputs for solenoid-actuated devices (e.g., reinforcer dispensers). The large number of low-current outputs is desirable for experiments that require many different visual displays, such as matching-to-sample studies. Up to 16 different types of responses from the subject can be processed through the PIO port, and these responses are debounced and encoded (letters A-Q) by software resident in the memory of the interface.

The memory in the interface consists of $1 \mathrm{~K}$ of RAM and $1 \mathrm{~K}$ of ROM, with space available for $4 \mathrm{~K}$. The RAM memory temporarily stores incoming information from the subject until 192 locations are filled, at which time a program in ROM dumps the data onto a single line emanating from the PIO port. This line is attached to a PET-compatible audio cassette recorder (or the PET's cassette recorder) and contains wires for turning the cassette motor on and off, as well as wires for transmitting data to the record head of this recorder. This operation is totally automatic and takes about 5 sec. During this interval, incoming data are stored in another part of RAM. The remaining space in ROM is devoted to utility programs that communicate with the PET and the PIO port, decode the special interface commands sent to the interface by the PET, monitor and control the interface's real-time clock, and debounce 
Table 1

Interface Macro-Command Set

\begin{tabular}{ll}
\hline \multicolumn{1}{c}{ Command } & \multicolumn{1}{c}{ Function } \\
\hline ST & Set Timer-Sets timer to hours, minutes, and seconds; zeroes the jiffy register. \\
DT & Display Time-Reads time (hours, minutes, seconds, and jiffies) into string. \\
EL_- & Energize Lamp (1-42). \\
DL & Deenergize Lamp (1-42). \\
ES & Energize Solenoid (1-6). \\
DS_ & Deenergize Solenoid (1-6). \\
CL & Clear Lamps-Turns all lamps and solenoids off. \\
BT_-- & Begin Test-Tape header with 1-16 characters supplied by user to identify name of tape. \\
ET & End Test-Puts trailer on tape after dumping remaining data from interface buffer. \\
ER_- & Enable Responses-All responses go into one of three 192-byte buffers in interface. \\
DR_- & Disable Responses-Responses will not be accepted by interface. \\
IN & Initialize-Allows User to enter information such as subject ID, date, session number. \\
\hline
\end{tabular}

Table 2

Program to Illustrate Use of PRINT and INPUT Commands

\begin{tabular}{lll}
\hline & Program & Explanation \\
100 & INPUT \#4, A\$ & Waits until a switch is closed. \\
200 & IF A $<>$ “C" GO TO 1000 & Was switch "C" closed? \\
300 & PRINT \#4, “EL4" & Yes, turn on Lamp 4-leaves it on. \\
400 & GO TO 1000 & \\
1000 & ROUTINE TO CHECK FOR ANOTHER SWITCH CLOSURE & \\
\hline
\end{tabular}

and encode the switch closures sent to the interface by the experimental subject.

The interface's timer (see Figure 2), which is a realtime clock driven by the $110-\mathrm{V}$ ac line frequency, presents data in the form of 8 bits for hours, minutes, seconds, and jiffies (hh, mm, ss, jj). This clock is used to time each event, whether input or output, and is written on the cassette tape along with the associated event. Note that the PET's timer, not the interface timer, is used to control time intervals in the program of experimental control (e.g., as in a fixed-interval schedule of reinforcement).

\section{Special Interface Commands}

Instead of using the BASIC commands PEEK or POKE to communicate with the intelligent interface, a simplified set of macro commands is available, as shown in Table 1.

In general, two BASIC commands are used to communicate with the interface, which is identified as Port 4. A PRINT \#4 command sends data to the interface, and an INPUT \#4 command accepts data from the interface. The normal state of the PET's program, when not performing some operation, is to listen to the interface by executing the INPUT \#4 command. The program in Table 2 illustrates the use of these commands in a program designed to detect whether Switch $\mathrm{C}$ was pressed, and if it was, then Light 4 is energized.

\section{Tape Format and Data Analysis}

When the 192-byte buffer in the interface is filled with data, the external cassette's motor is energized, and these data are automatically written on tape as ASCII string characters. Four types of information are placed in the interface data buffer: (1) tape header and initialization information (prior to first data record). (2) Activation record (what switch was pressed; format is "C, _ _ _ _ , where $\mathrm{C}$ indicates switch pressed, followed by an eight-digit number to indicate the hours, minutes, seconds, and jiffies at the time Switch $C$ was pressed in real-time). (3) Activity record (command sent to interface; format is "DL5, ___ _ , " which indicates that Light 5 was turned off at time indicated by the following eight-digit real-time number). (4) Trailer-end of session (following last data record on tape).

To perform data analysis, the tape can be read by any PET, but preferably by a PET with the maximum memory, $32 \mathrm{~K}$, so that, if necessary, all data can be read into the PET's memory before processing. It is possible to process portions of the data by reading and analyzing each portion separately and then combining the results, but the program to handle such analysis is more complicated to write and takes longer. It is also advisable to have a printer attached to the PET used for data analysis, since a hard copy of the data provides back-up should a data cassette tape be accidentally erased.

The use of low-cost cassette tape to store data presents an attractive medium for archival storage and makes possible the swapping of raw data among investigators with similar interests and access to a PET computer.

\section{REFERENCES}

Crossman, E. K., \& Williams, J. G. A multi-user on-line 8080 microcomputer system. Behavior Research Methods \& Instrumentation, 1978, 10, 254-258.

McLean, R. S. The Commodore PET: Using computers for experimental control. Behavior Research Methods \& Instrumentation, 1978, 10, 468-473. 